\title{
Comprehensive analysis of IncRNA-associated ceRNA network reveals the novel potential of IncRNA, miRNA and mRNA biomarkers in human rectosigmoid junction cancer
}

\author{
QIANSHI ZHANG ${ }^{1 *}$, ZHEN FENG $^{1 *}$, SHASHA SHI $^{2}$, YU ZHANG $^{3}$ and SHUANGYI REN ${ }^{1}$ \\ Departments of ${ }^{1}$ Gastrointestinal Surgery, ${ }^{2}$ Ultrasound and ${ }^{3}$ Clinical Laboratory, \\ The Second Affiliated Hospital of Dalian Medical University, Dalian, Liaoning 116023, P.R. China
}

Received March 11, 2020; Accepted November 20, 2020

DOI: $10.3892 / 01.2020 .12405$

\begin{abstract}
Although accumulating evidence has confirmed the potential biological functions of long non-coding RNAs (lncRNAs) as competitive endogenous RNAs (ceRNAs) in colorectal tumorigenesis and progression, few studies have focused on rectosigmoid junction cancer. In the present study, a comprehensive analysis was conducted to explore lncRNA-mediated ceRNA implications and their potential value for prognosis. IncRNA, microRNA (miR/miRNA) and mRNA expression profiles were downloaded from The Cancer Genome Atlas database. Subsequently, a IncRNA-miRNA-mRNA regulatory network was constructed to evaluate the functions of these differentially expressed genes on overall survival (OS) for rectosigmoid junction cancer. As a result, a rectosigmoid junction cancer-specific ceRNA network was successfully constructed with 7 differentially expressed (DE)lncRNAs, 16 DEmiRNAs and 71 DEmRNAs. Among the network, one DElncRNA (small nucleolar RNA host
\end{abstract}

Correspondence to: $\mathrm{Dr} \mathrm{Yu}$ Zhang, Department of Clinical Laboratory, The Second Affiliated Hospital of Dalian Medical University, 467 Zhongshan Road, Dalian, Liaoning 116023, P.R. China

E-mail: 735881526@qq.com

Professor Shuangyi Ren, Department of Gastrointestinal Surgery, The Second Affiliated Hospital of Dalian Medical University, 467 Zhongshan Road, Dalian, Liaoning 116023, P.R. China

E-mail: rsydl@aliyun.com

*Contributed equally

Abbreviations: lncRNA, long non-coding RNA; ceRNA, competitive endogenous RNA; TCGA, The Cancer Genome Atlas; miRNA, microRNA; OS, overall survival; DE, differentially expressed; CRC, colorectal cancer; MREs, miRNA response elements; KEGG, Kyoto Encyclopedia of Genes and Genomes; FC, fold-change

Key words: CRC, rectosigmoid junction, ceRNA, bioinformatics analysis, TCGA gene 20) and three mRNAs (sodium- and chloride-dependent taurine transporter, fibroblast growth factor 13 and tubulin polyglutamylase TTLL7) were significantly associated with OS $(\mathrm{P}<0.05)$. Additionally, two lncRNAs (KCNQ1OT1 and MIR17HG) interacted with most of the DEmiRNAs. Notably, two top-ranked miRNAs (hsa-miR-374a-5p and hsa-miR-374b-5p) associated networks were identified to be markedly associated with the pathogenesis. Furthermore, four DEmRNAs (caveolin-1, MET, filamin-A and AKT3) were enriched in the Kyoto Encylopedia of Gene and Genomes pathway analysis, as well as being included in the ceRNA network. In summary, the present results revealed that a specific IncRNA-miRNA-mRNA network was associated with rectosigmoid junction cancer, providing several molecules that may be used as novel prognostic biomarkers and therapeutic targets.

\section{Introduction}

Rectosigmoid junction cancer accounts for $9 \%$ of colorectal cancer (CRC) cases (1), ranking third in terms of incidence $(10.2 \%)$ and with the second-highest mortality rate $(9.2 \%)$ worldwide in 2018 (2). The rectosigmoid junction (C19.9) is encoded as an independent segment of the intesimum crassum in the International Classification of Disease for Oncology of the World Health Organization (3).

To the best of our knowledge, few studies have discussed rectosigmoid junction cancer, in which the rectosigmoid junction was not entirely researched separately and was either included in the colon or the rectum (4-7). Meanwhile, the treatment of colorectal tumors has become increasingly differentiated and individualized. Hence, there is an urgent need of identifying effective potential molecular biomarkers for the early diagnosis, prognosis and treatment of rectosigmoid junction cancer.

In the last decade, regulatory networks composed of long non-coding RNAs (lncRNAs), microRNAs (miRs/miRNAs) and mRNAs have stimulated great interest in the research of molecular biological mechanisms participating in the pathogenesis and prognosis of tumors (8-10). The ceRNA theory was proposed by Salmena et al (11) in 2011, which established the existence of a complex post-transcriptional regulatory network, 
where lncRNAs, mRNAs and other non-coding RNAs share mutual miRNA response elements (MREs) with miRNAs, and that ceRNAs can act as natural miRNA sponges to suppress miRNA function by competitively binding to MREs on the target mRNA. In the ceRNA network, lncRNAs function as ceRNAs to adjust the level of protein-coding genes and take part in the regulation of cell biology by sponging miRNAs (11).

In recent years, IncRNA-mediated ceRNA regulatory networks were proven to be implicated in the tumorigenesis and progression of CRC (12-17). Nevertheless, to the best of our knowledge, a comprehensive analysis of the rectosigmoid junction cancer-associated lncRNA-mediated ceRNA network has not been performed.

The present study compared integrated RNA expression profiles based on The Cancer Genome Atlas (TCGA) database between 69 tumor tissues and 7 normal tissues of rectosigmoid junction cancer. Next, an integrated analysis was performed to select aberrantly expressed lncRNAs, miRNAs and mRNAs. Finally, 9 lncRNAs, 16 miRNAs and 71 mRNAs were screened to build the lncRNA-miRNA-mRNA ceRNA network. Furthermore, relevant overall survival (OS) analysis of differentially expressed (DE)RNAs involved in the ceRNA network was performed to screen significant prognostic biomarkers.

\section{Materials and methods}

Sequencing and clinical data. The RNA expression and corresponding clinical data of patients with rectosigmoid junction cancer embedded in the comprehensive analysis were obtained from the TCGA database (https://portal.gdc.cancer.gov/). The following exclusion criteria were used: i) Patients suffering malignancies other than rectosigmoid junction cancer; ii) samples with incomplete data for analysis; iii) duplicate samples; and iv) patients who underwent preoperative neoadjuvant radiotherapy and chemotherapy. Overall, data from a total of 69 tumor tissues and 7 normal tissues adjacent to surgically removed tumors were obtained from 69 patients with rectosigmoid junction cancer. The patients consisted of 35 males and 34 females, with a mean age of 63.9 years and a median age of 67 years (age range, 31-90 years). The RNA and miRNA sequence data from the 76 cases originated from Illumina HiSeq2000 and Illumina Genome Analyzer IIX platforms. Their detailed information is shown in Table SI. The Union for International Cancer Control/American Joint Committee on Cancer TNM staging system was used (18).

Aberrant expression analysis. Multiple data, including sequencing and clinical data, were analyzed by running the 'gdcDEAnalysis' package in R-Portable language software (v3.5.1; https://www.r-project.org/). Adjusted $\mathrm{P}<0.01$ and $\log 2$ [fold-change $(\mathrm{FC})]>2.0$ were set as statistical significance for DElncRNAs and DEmRNAs, while $\log _{2}(\mathrm{FC})>2.5$ and adjusted $\mathrm{P}<0.01$ were considered for DEmiRNAs. LogFC $>0$ was set to high expression, and $\log \mathrm{FC}<0$ was set to low expression. For the screening of DElncRNAs, DEmiRNAs and DEmRNAs, volcano maps were generated using the 'gdcVolcanoPlot' package (v1.1.1) in R.

Functional enrichment analysis. The Gene Ontology (GO) analysis and the Kyoto Encyclopedia of Genes and
Genomes (KEGG) pathway analysis of DEmRNAs were performed using the $\mathrm{R}$ platform. A KEGG pathway or GO term with a false discovery rate (FDR) $<0.01$ was considered statistically significant. The GO analysis of the cellular component (CC), molecular function (MF) and biological process (BP), and the enriched pathways of DEmRNAs were ranked by enrichment score $\left[-\log _{10}(\mathrm{P}\right.$-value $\left.)\right]$ and performed to investigate the underlying function of all DEmRNAs.

Formation of the lncRNA-miRNA-mRNA network. The IncRNA-miRNA-mRNA ceRNA network was based on the hypothesis that lncRNAs can directly interact with miRNA sponges to influence the bioactivity of mRNAs (11). The ceRNA network of rectosigmoid junction cancer was established as follows.

Firstly, highly reliable miRNA reference databases [mirTarBase (19) (http://mirtarbase.mbc.nctu.edu.tw/php/index. php), miRcode (http://www.mircode.org/) and ENCORI (20) (http://starbase.sysu.edu.cn/)] were utilised to determine miRNA-targeted mRNAs. Subsequently, miRNA-targeted lncRNAs were retrieved from SpongeScan (http://spongescan. rc.ufl.edu/), miRcode and ENCORI databases. The intersection of the overlapping predicted results, DElncRNAs and DEmRNAs was then obtained. Furthermore, the miRNAs negatively regulating the intersection expression of lncRNAs and mRNAs were selected based on the aforementioned ceRNA hypothesis. The IncRNA-miRNA-mRNA network was visualized using Cytoscape v3.6.1 (https://cytoscape.org/). The process of ceRNA network construction is shown in the flowchart presented in Fig. 1.

Generation of functional lncRNA-miRNA-mRNA regulatory modules. In order to screen the functional lncRNA-miRNA-mRNA regulatory modules of rectosigmoid junction cancer, the degree, Maximal Clique Centrality (MCC), betweenness centrality and closeness centrality of lncRNAs were calculated using the cytoHubba plugin on Cytoscape v3.6.1. The overlapping top-ranked lncRNAs among the four calculation methods were extracted.

Survival analysis of candidate RNAs. To identify prognostic DERNA signatures, the survival curves of all RNAs involved in the ceRNA network were plotted using the R survival package (v1.1.1). Univariate survival analysis was estimated based on the Kaplan-Meier curve analysis using the log-rank test. Unless otherwise stated, $\mathrm{P}<0.05$ was considered to indicate a statistically significant difference.

\section{Results}

Identification of DElncRNAs. A total of 151 DElncRNAs were identified by running the 'gdcDEAnalysis' package in R. As a result, 109 (72.19\%) upregulated and 42 (27.81\%) downregulated DElncRNAs were identified (Fig. 2A). The top 21 upregulated and 21 downregulated lncRNAs, and the related $\operatorname{logFC}, \mathrm{P}$-values and FDR values, are listed in Table I.

Identification of DEmiRNAs. A total of 243 DEmiRNAs were identified using the 'gdcDEAnalysis' package in R. As shown in Fig. 2B, there were $155(63.79 \%)$ upregulated and 88 (36.21\%) downregulated DEmiRNAs. The top 21 upregulated and 


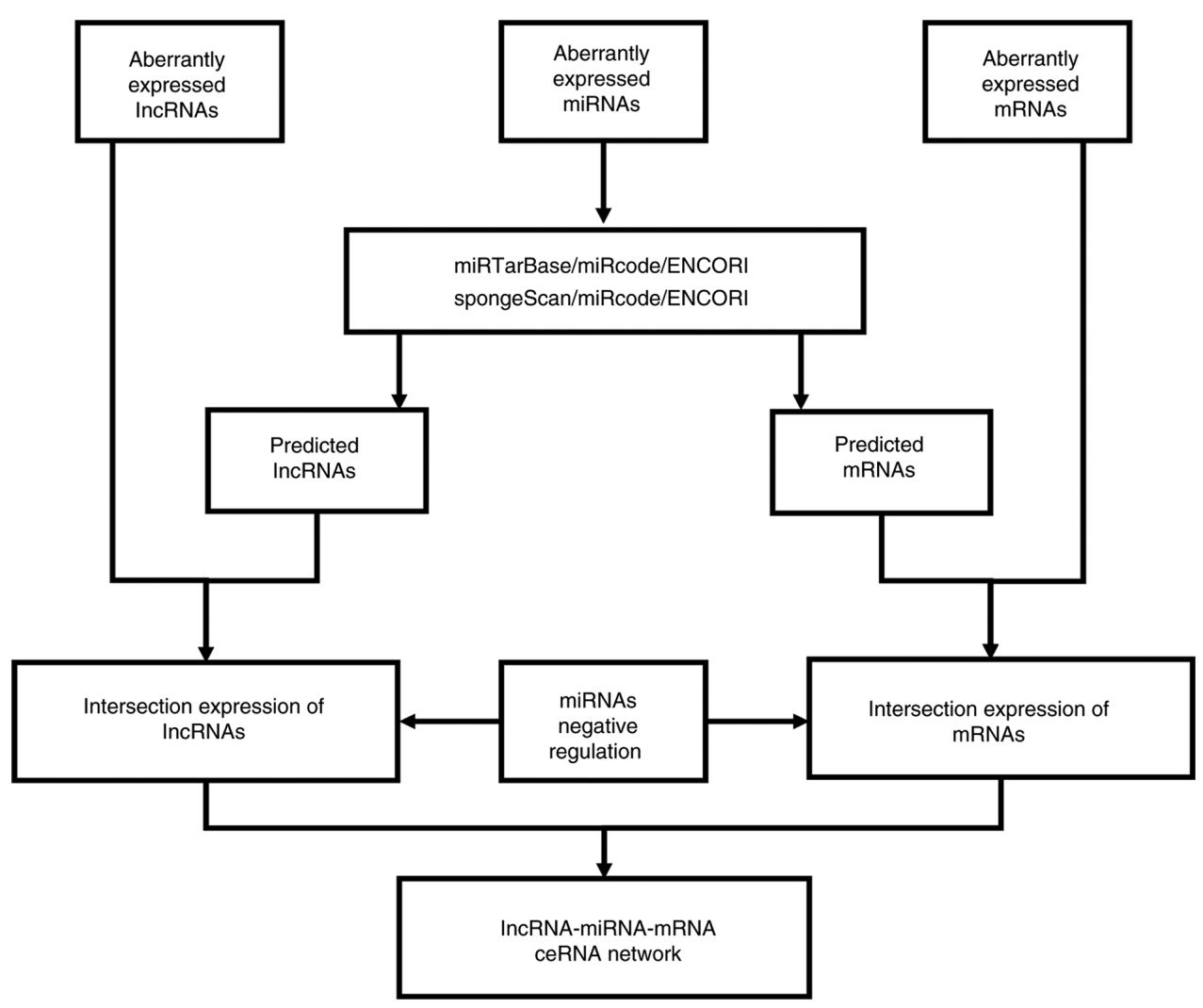

Figure 1. ceRNA network analysis flowchart. ceRNA, competitive endogenous RNA; miRNA, microRNA; lncRNA, long non-coding RNA.
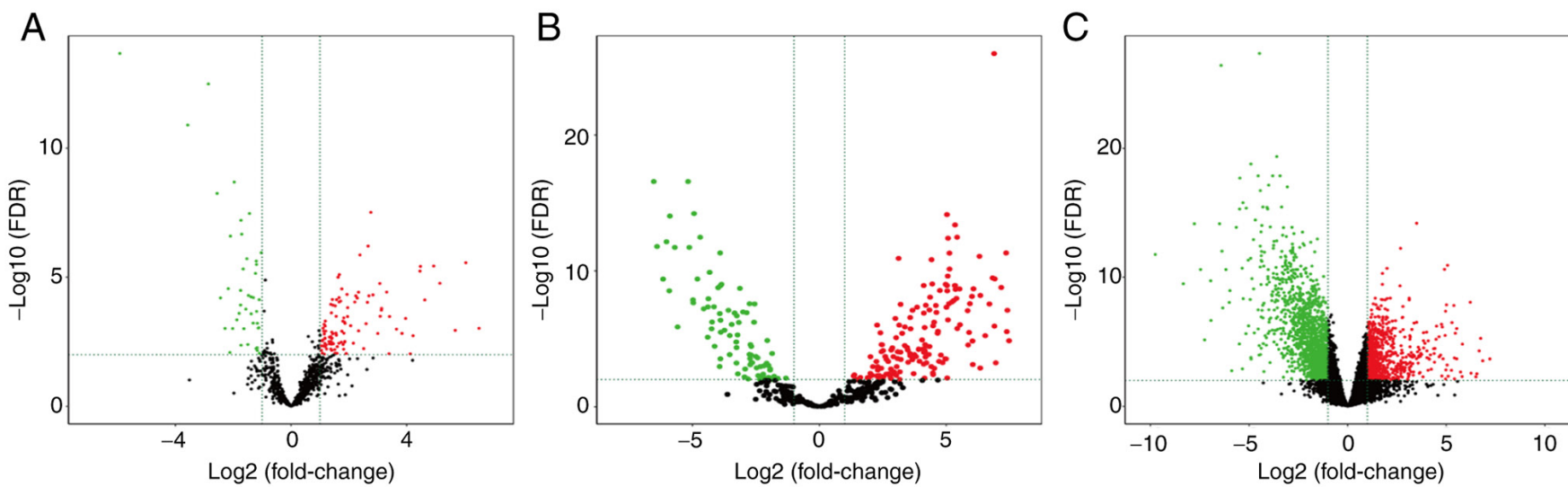

Figure 2. Volcano plots of differentially expressed genes. Differentially expressed (A) long non-coding RNAs, (B) microRNAs and (C) mRNAs. Volcano plots were constructed using $\log _{2}$ (fold-change) $\mid>2.0$ and FDR $<0.01$. Green dots represent downregulated differentially expressed genes, while red dots represent upregulated differentially expressed genes. FDR, false discovery rate.

21 downregulated lncRNAs, and the related $\log F C$, P-values and FDR values are listed in Table II.

Identification of DEmRNAs. A total of 2,231 DEmRNAs were selected using the 'gdcDEAnalysis' package. As shown in Fig. 2C, 793 (35.54\%) DEmRNAs were upregulated and 1,438 (64.46\%) DEmRNAs were downregulated. The top 21 upregulated and 21 downregulated lncRNAs, and the related $\log$ FC, $\mathrm{P}$-values and FDR values are listed in Table III.

GO functional enrichment and KEGG pathway analysis of DEmRNAs. GO analysis indicated significant enrichment in the BP of 'leukocyte migration', 'positive regulation of cytosolic calcium ion concentration' and 'regulation of chemotaxis'. CC analysis suggested enrichment in the 'proteinaceous extracellular matrix', 'sarcolemma' and 'costamere'. Additionally, the DEmRNAs exhibited MF enrichment in 'transcription factor activity, RNA polymerase II proximal promoter sequence-specific DNA binding' (Fig. 3A).

KEGG pathway analysis allows for the functional annotation of DEmRNAs. The present study obtained nine KEGG pathways. The dotplot in Fig. 3B shows the results of the KEGG pathway with P-values ranging from low to high. The complete results of the KEGG pathway analysis are shown in Table IV. 
Table I. Top 21 upregulated and 21 downregulated differentially expressed long non-coding RNAs in rectosigmoid junction cancer samples.

A, Upregulated

\begin{tabular}{lccc}
\hline Name & $\log (\mathrm{FC})$ & P-value & FDR \\
\hline MAFG-AS1 & 2.752340839 & $6.67 \times 10^{-10}$ & $3.10 \times 10^{-08}$ \\
PVT1 & 2.657857958 & $2.18 \times 10^{-08}$ & $6.25 \times 10^{-07}$ \\
SNHG17 & 2.378579537 & $5.72 \times 10^{-08}$ & $1.39 \times 10^{-06}$ \\
LINC02253 & 6.040229343 & $1.30 \times 10^{-07}$ & $2.77 \times 10^{-06}$ \\
BLACAT1 & 4.927333574 & $1.84 \times 10^{-07}$ & $3.72 \times 10^{-06}$ \\
AC021218.1 & 4.467747207 & $1.93 \times 10^{-07}$ & $3.85 \times 10^{-06}$ \\
MGC32805 & 4.452660405 & $3.21 \times 10^{-07}$ & $5.85 \times 10^{-06}$ \\
SNHG15 & 1.667019645 & $4.62 \times 10^{-07}$ & $8.02 \times 10^{-06}$ \\
CAPN10-AS1 & 1.622484665 & $6.05 \times 10^{-07}$ & $1.01 \times 10^{-05}$ \\
CASC21 & 5.142108745 & $1.14 \times 10^{-06}$ & $1.73 \times 10^{-05}$ \\
LINC01605 & 3.06645948 & $1.16 \times 10^{-06}$ & $1.76 \times 10^{-05}$ \\
LINC01232 & 1.751414603 & $2.04 \times 10^{-06}$ & $2.84 \times 10^{-05}$ \\
CRNDE & 3.301683337 & $2.89 \times 10^{-06}$ & $3.79 \times 10^{-05}$ \\
AL121832.3 & 2.327872764 & $2.93 \times 10^{-06}$ & $3.84 \times 10^{-05}$ \\
MIR4435-2HG & 1.932326125 & $3.66 \times 10^{-06}$ & $4.68 \times 10^{-05}$ \\
MIR17HG & 2.827330119 & $3.84 \times 10^{-06}$ & $4.86 \times 10^{-05}$ \\
VPS9D1-AS1 & 2.686552396 & $4.25 \times 10^{-06}$ & $5.29 \times 10^{-05}$ \\
AC073335.2 & 2.234963344 & $4.26 \times 10^{-06}$ & $5.29 \times 10^{-05}$ \\
ZFAS1 & 1.656374536 & $5.13 \times 10^{-06}$ & $6.19 \times 10^{-05}$ \\
FOXP4-AS1 & 2.284408492 & $5.42 \times 10^{-06}$ & $6.49 \times 10^{-05}$ \\
SNHG20 & 1.424697676 & $6.31 \times 10^{-06}$ & $7.32 \times 10^{-05}$ \\
\hline & & & \\
& & & \\
\hline
\end{tabular}

B, Downregulated

\begin{tabular}{lccc}
\hline Name & $\log (\mathrm{FC})$ & P-value & FDR \\
\hline CDKN2B-AS1 & -5.926520656 & $4.05 \times 10^{-17}$ & $2.15 \times 10^{-14}$ \\
MBNL1-AS1 & -2.861799886 & $9.56 \times 10^{-16}$ & $3.30 \times 10^{-13}$ \\
HAGLR & -3.577536261 & $6.83 \times 10^{-14}$ & $1.28 \times 10^{-11}$ \\
AC016027.1 & -1.97046862 & $2.68 \times 10^{-11}$ & $2.06 \times 10^{-09}$ \\
AC106869.1 & -2.561290755 & $9.22 \times 10^{-11}$ & $5.72 \times 10^{-09}$ \\
PDCD4-AS1 & -1.440015713 & $7.53 \times 10^{-10}$ & $3.44 \times 10^{-08}$ \\
MIR22HG & -1.735841168 & $1.49 \times 10^{-09}$ & $6.29 \times 10^{-08}$ \\
B4GALT1-AS1 & -1.711349731 & $6.23 \times 10^{-09}$ & $2.17 \times 10^{-07}$ \\
ZNF667-AS1 & -2.1010172 & $7.62 \times 10^{-09}$ & $2.57 \times 10^{-07}$ \\
LINC00294 & -1.035031181 & $4.44 \times 10^{-08}$ & $1.14 \times 10^{-06}$ \\
LINC00641 & -1.533058923 & $8.46 \times 10^{-08}$ & $1.94 \times 10^{-06}$ \\
AL691432.2 & -1.206788789 & $1.09 \times 10^{-07}$ & $2.39 \times 10^{-06}$ \\
AC006333.2 & -1.201587737 & $1.57 \times 10^{-07}$ & $3.25 \times 10^{-06}$ \\
ZNF710-AS1 & -1.66124648 & $2.57 \times 10^{-07}$ & $4.85 \times 10^{-06}$ \\
U91328.2 & -1.235272793 & $4.09 \times 10^{-07}$ & $7.20 \times 10^{-06}$ \\
FENDRR & -2.175974452 & $2.00 \times 10^{-06}$ & $2.79 \times 10^{-05}$ \\
AC016888.1 & -1.749061747 & $2.42 \times 10^{-06}$ & $3.27 \times 10^{-05}$ \\
AL135905.2 & -1.476920272 & $4.08 \times 10^{-06}$ & $5.13 \times 10^{-05}$ \\
AP002761.4 & -1.384651324 & $4.75 \times 10^{-06}$ & $5.79 \times 10^{-05}$ \\
AL662844.4 & -1.308823325 & $5.14 \times 10^{-06}$ & $6.19 \times 10^{-05}$ \\
LINC02441 & -2.443144586 & $5.33 \times 10^{-06}$ & $6.38 \times 10^{-05}$ \\
\hline
\end{tabular}

FC, fold-change; FDR, false discovery rate.
Table II. Top 21 upregulated and 21 downregulated differentially expressed miRNAs in rectosigmoid junction cancer samples.

A, Upregulated

\begin{tabular}{lccc}
\hline Name & $\log (\mathrm{FC})$ & P-value & FDR \\
\hline hsa-miR-21-5p & 6.884706944 & $2.22 \times 10^{-29}$ & $1.01 \times 10^{-26}$ \\
hsa-miR-126-3p & 5.027647848 & $9.38 \times 10^{-17}$ & $7.12 \times 10^{-15}$ \\
hsa-miR-101-3p & 5.347152405 & $7.17 \times 10^{-16}$ & $4.08 \times 10^{-14}$ \\
hsa-miR-106b-5p & 5.429063826 & $7.18 \times 10^{-15}$ & $3.27 \times 10^{-13}$ \\
hsa-miR-24-2-5p & 5.070084953 & $9.31 \times 10^{-15}$ & $3.85 \times 10^{-13}$ \\
hsa-miR-452-5p & 7.358520015 & $1.68 \times 10^{-13}$ & $4.71 \times 10^{-12}$ \\
hsa-miR-192-5p & 5.126016035 & $1.92 \times 10^{-13}$ & $4.85 \times 10^{-12}$ \\
hsa-miR-141-3p & 6.308116527 & $3.48 \times 10^{-13}$ & $8.33 \times 10^{-12}$ \\
hsa-miR-24-3p & 3.122933649 & $5.22 \times 10^{-13}$ & $1.19 \times 10^{-11}$ \\
hsa-miR-194-5p & 4.418901392 & $6.84 \times 10^{-13}$ & $1.48 \times 10^{-11}$ \\
hsa-miR-379-5p & 5.130530865 & $3.49 \times 10^{-12}$ & $7.22 \times 10^{-11}$ \\
hsa-miR-889-3p & 5.058722699 & $1.26 \times 10^{-11}$ & $2.38 \times 10^{-10}$ \\
hsa-miR-429 & 6.825921399 & $1.78 \times 10^{-11}$ & $3.24 \times 10^{-10}$ \\
hsa-miR-19b-3p & 6.910550025 & $2.17 \times 10^{-11}$ & $3.80 \times 10^{-10}$ \\
hsa-miR-130b-3p & 4.447213787 & $5.73 \times 10^{-11}$ & $8.99 \times 10^{-10}$ \\
hsa-miR-140-5p & 4.976522784 & $6.45 \times 10^{-11}$ & $9.47 \times 10^{-10}$ \\
hsa-miR-16-5p & 5.352104156 & $9.06 \times 10^{-11}$ & $1.17 \times 10^{-09}$ \\
hsa-miR-141-5p & 5.336691795 & $8.98 \times 10^{-11}$ & $1.17 \times 10^{-09}$ \\
hsa-miR-200a-3p & 5.028635315 & $9.22 \times 10^{-11}$ & $1.17 \times 10^{-09}$ \\
hsa-miR-26b-5p & 4.109853806 & $9.13 \times 10^{-11}$ & $1.17 \times 10^{-09}$ \\
hsa-miR-374a-3p & 7.166807838 & $1.32 \times 10^{-10}$ & $1.63 \times 10^{-09}$ \\
\hline
\end{tabular}

B, Downregulated

\begin{tabular}{lccc}
\hline Name & $\log (\mathrm{FC})$ & P-value & FDR \\
\hline hsa-miR-766-3p & -5.176574242 & $2.30 \times 10^{-19}$ & $2.61 \times 10^{-17}$ \\
hsa-miR-197-3p & -5.178244912 & $2.09 \times 10^{-19}$ & $2.61 \times 10^{-17}$ \\
hsa-miR-328-3p & -6.527470364 & $2.25 \times 10^{-19}$ & $2.61 \times 10^{-17}$ \\
hsa-let-7d-3p & -4.940073713 & $6.56 \times 10^{-17}$ & $5.97 \times 10^{-15}$ \\
hsa-miR-139-5p & -5.893898996 & $1.40 \times 10^{-16}$ & $9.09 \times 10^{-15}$ \\
hsa-miR-1306-5p & -4.697991354 & $6.86 \times 10^{-15}$ & $3.27 \times 10^{-13}$ \\
hsa-miR-6511b-3p & -6.027857145 & $1.86 \times 10^{-14}$ & $7.07 \times 10^{-13}$ \\
hsa-miR-129-5p & -6.398340279 & $4.50 \times 10^{-14}$ & $1.57 \times 10^{-12}$ \\
hsa-miR-574-3p & -5.128565486 & $6.02 \times 10^{-14}$ & $1.83 \times 10^{-12}$ \\
hsa-miR-149-5p & -5.701365419 & $5.87 \times 10^{-14}$ & $1.83 \times 10^{-12}$ \\
hsa-miR-99b-5p & -3.912360633 & $1.76 \times 10^{-13}$ & $4.71 \times 10^{-12}$ \\
hsa-miR-195-3p & -4.325681902 & $6.36 \times 10^{-12}$ & $1.26 \times 10^{-10}$ \\
hsa-miR-125a-5p & -4.807276424 & $2.32 \times 10^{-11}$ & $3.91 \times 10^{-10}$ \\
hsa-miR-139-3p & -6.158797314 & $2.43 \times 10^{-11}$ & $3.94 \times 10^{-10}$ \\
hsa-let-7e-3p & -3.856910446 & $6.25 \times 10^{-11}$ & $9.47 \times 10^{-10}$ \\
hsa-miR-1976 & -3.895162028 & $9.09 \times 10^{-11}$ & $1.17 \times 10^{-09}$ \\
hsa-miR-378a-5p & -3.988573695 & $1.40 \times 10^{-10}$ & $1.68 \times 10^{-09}$ \\
hsa-let-7b-3p & -3.134133079 & $1.61 \times 10^{-10}$ & $1.88 \times 10^{-09}$ \\
hsa-miR-486-5p & -5.916429249 & $2.81 \times 10^{-10}$ & $2.85 \times 10^{-09}$ \\
hsa-miR-193a-5p & -4.373097741 & $1.21 \times 10^{-09}$ & $1.10 \times 10^{-08}$ \\
hsa-miR-642a-5p & -4.982818274 & $1.48 \times 10^{-09}$ & $1.32 \times 10^{-08}$ \\
& & & \\
\hline
\end{tabular}

miR/miRNA, microRNA; FC, fold-change; FDR, false discovery rate. 
Table III. Top 21 upregulated and 21 downregulated differentially expressed mRNAs in rectosigmoid junction cancer samples.

A, Upregulated

\begin{tabular}{lccc}
\hline Name & $\log ($ FC $)$ & P-value & FDR \\
\hline TGFBI & 3.490648701 & $8.74 \times 10^{-18}$ & $6.49 \times 10^{-15}$ \\
NFE2L3 & 2.676746374 & $1.85 \times 10^{-15}$ & $5.72 \times 10^{-13}$ \\
ETV4 & 5.060920019 & $6.19 \times 10^{-14}$ & $1.19 \times 10^{-11}$ \\
NEBL & 1.988240629 & $1.25 \times 10^{-13}$ & $2.06 \times 10^{-11}$ \\
CDH3 & 4.902363409 & $1.52 \times 10^{-13}$ & $2.45 \times 10^{-11}$ \\
GRAMD1A & 1.756550954 & $3.72 \times 10^{-13}$ & $5.09 \times 10^{-11}$ \\
KAT2A & 1.753706981 & $3.56 \times 10^{-12}$ & $3.55 \times 10^{-10}$ \\
FUT1 & 2.650628542 & $4.84 \times 10^{-12}$ & $4.67 \times 10^{-10}$ \\
GTF2IRD1 & 2.158124288 & $5.98 \times 10^{-11}$ & $4.17 \times 10^{-09}$ \\
CARD14 & 3.115786643 & $6.05 \times 10^{-11}$ & $4.18 \times 10^{-09}$ \\
DKC1 & 1.389511094 & $6.06 \times 10^{-11}$ & $4.18 \times 10^{-09}$ \\
SOX9 & 1.996396591 & $6.43 \times 10^{-11}$ & $4.41 \times 10^{-09}$ \\
CBX8 & 1.700207115 & $8.05 \times 10^{-11}$ & $5.24 \times 10^{-09}$ \\
ENC1 & 1.754690772 & $8.47 \times 10^{-11}$ & $5.42 \times 10^{-09}$ \\
SLC6A6 & 3.116405062 & $9.10 \times 10^{-11}$ & $5.70 \times 10^{-09}$ \\
VEGFA & 1.894191494 & $9.10 \times 10^{-11}$ & $5.70 \times 10^{-09}$ \\
KRT80 & 6.211404628 & $1.52 \times 10^{-10}$ & $8.69 \times 10^{-09}$ \\
XPO5 & 1.289131767 & $2.15 \times 10^{-10}$ & $1.17 \times 10^{-08}$ \\
NOP2 & 1.428528716 & $2.53 \times 10^{-10}$ & $1.35 \times 10^{-08}$ \\
SALL4 & 5.004008301 & $2.62 \times 10^{-10}$ & $1.38 \times 10^{-08}$ \\
STPG4 & 5.379593583 & $3.00 \times 10^{-10}$ & $1.55 \times 10^{-08}$ \\
\hline
\end{tabular}

B, Downregulated

\begin{tabular}{llll}
\hline Name & \multicolumn{1}{l}{$\log (\mathrm{FC})$} & P-value & FDR \\
\hline CLEC3B & -4.476185728 & $2.90 \times 10^{-32}$ & $4.31 \times 10^{-28}$ \\
BEST4 & -6.422652094 & $4.95 \times 10^{-31}$ & $3.67 \times 10^{-27}$ \\
GCNT2 & -3.601840574 & $8.79 \times 10^{-24}$ & $4.34 \times 10^{-20}$ \\
LYVE1 & -4.911974763 & $4.40 \times 10^{-23}$ & $1.63 \times 10^{-19}$ \\
FAM107A & -3.43110438 & $6.40 \times 10^{-22}$ & $1.36 \times 10^{-18}$ \\
LIFR & -3.8063879 & $5.06 \times 10^{-22}$ & $1.36 \times 10^{-18}$ \\
ABCG2 & -4.544022652 & $5.74 \times 10^{-22}$ & $1.36 \times 10^{-18}$ \\
ABCA8 & -5.474165401 & $1.09 \times 10^{-21}$ & $2.02 \times 10^{-18}$ \\
GLDN & -4.008350765 & $4.32 \times 10^{-21}$ & $7.12 \times 10^{-18}$ \\
GNG7 & -3.06421636 & $6.59 \times 10^{-21}$ & $9.78 \times 10^{-18}$ \\
PKIB & -4.432199222 & $1.41 \times 10^{-20}$ & $1.90 \times 10^{-17}$ \\
MT1M & -5.317272589 & $1.35 \times 10^{-19}$ & $1.67 \times 10^{-16}$ \\
RNF152 & -3.354536011 & $3.38 \times 10^{-19}$ & $3.58 \times 10^{-16}$ \\
SCN9A & -4.300397577 & $3.17 \times 10^{-19}$ & $3.58 \times 10^{-16}$ \\
C20rf88 & -4.094084525 & $3.87 \times 10^{-19}$ & $3.82 \times 10^{-16}$ \\
ALPI & -5.148481336 & $4.75 \times 10^{-19}$ & $4.40 \times 10^{-16}$ \\
SULT1A2 & -4.06180475 & $6.24 \times 10^{-19}$ & $5.15 \times 10^{-16}$ \\
SFRP1 & -5.485109328 & $6.03 \times 10^{-19}$ & $5.15 \times 10^{-16}$ \\
TNXB & -4.688553804 & $4.63 \times 10^{-18}$ & $3.61 \times 10^{-15}$ \\
ADH1B & -6.502588649 & $1.03 \times 10^{-17}$ & $7.12 \times 10^{-15}$ \\
AQP8 & -7.780183426 & $1.06 \times 10^{-17}$ & $7.12 \times 10^{-15}$ \\
\hline
\end{tabular}

FC, fold-change; FDR, false discovery rate.
Only one cancer-associated pathway, 'Proteoglycans in cancer', was identified, which contained 43 genes (Table SII) and a P-value of 0.007893 . This signaling pathway became the focus of subsequent analyses.

Construction of the ceRNA network in rectosigmoid junction cancer. To further understand the role of DElncRNAs in rectosigmoid junction cancer, the interaction of DEmiRNAs with DElncRNAs and DEmRNAs was predicted. A total of 98 DElncRNAs were predicted to interact with 16 DEmiRNAs using SpongeScan, miRcode and ENCORI databases. Furthermore, targeted mRNAs that were searched on the basis of the aforementioned 16 miRNAs were retrieved from mirTarBase, miRcode and ENCORI. A total of 1,411 targeted DEmRNAs that were selected were included in all three databases. The detailed information is shown in Table SIII.

Finally, 7 DElncRNAs, 16 DEmiRNAs and 71 DEmRNAs were screened to construct the ceRNA network. The association between DElncRNAs and DEmiRNAs is shown in Table V. All three types of RNAs that participated in constructing the IncRNA-miRNA-mRNA ceRNA network are listed in Table VI. The ceRNA regulatory network was visualized using Cytoscape software (Fig. 4).

Functional lncRNA-miRNA-mRNA regulatory modules. To specify the key lncRNA-miRNA-mRNA regulatory modules in the process of rectosigmoid junction cancer, the degree, betweenness centrality, MCC and closeness centrality were calculated using cytoHubba plugin on Cytoscape. The results of the top 10 RNAs calculated by the four aforementioned methods are listed in Table VII. The upregulated lncRNAs KCNQ1OT1 and MIR17HG were closely associated with most of the DEmiRNAs in the ceRNA network. In addition, the upregulated KCNQ1OT1 competed with five key DEmiRNAs (miR-152-3p, miR-148a-3p, miR-29c-3p, miR-29b-3p and miR-326) to regulate the expression of target genes. Two miRNAs, hsa-miR-374a-5p and hsa-miR-374b-5p, ranked top among all four calculation methods. The genes caveolin-1 (CAV-1), MET, filamin-A (FLNA) and AKT3 were enriched in the KEGG 'Proteoglycans in cancer' pathway, as well as being involved in the ceRNA network. This suggested that these two miRNAs and four mRNAs may serve essential roles in the carcinogenesis and development of rectosigmoid junction cancer.

Survival analysis for RNAs in the ceRNA network. To identify the potential RNAs with prognostic characteristics, Kaplan-Meier univariate analysis was applied to calculate the OS for all DElncRNAs, DEmiRNAs and DEmRNAs in rectosigmoid junction cancer. The results revealed that one DElncRNA and three mRNAs were significantly associated with OS $(\mathrm{P}<0.05)$. Furthermore, high expression levels of the only DElncRNA, small nucleolar RNA host gene 20 (SNHG20), and of one DEmRNA, sodium- and chloride-dependent taurine transporter (SLC6A6), were associated with a favourable prognosis, while low expression levels of the other two DEmRNAs [fibroblast growth factor 13 (FGF13) and tubulin polyglutamylase TTLL7 (TTLL7)] were associated with a favourable prognosis (Fig. 5). The OS data for DElncRNAs, DEmiRNAs and DEmRNAs are presented in Table SIV. 
Table IV. Kyoto Encyclopedia of Genes and Genomes pathways enriched in differentially expressed mRNAs in rectosigmoid junction cancer.

\begin{tabular}{llcc}
\hline PathwayID & \multicolumn{1}{c}{ Description } & P-value & Count \\
\hline hsa04020 & Calcium signaling pathway & $2.01 \times 10^{-06}$ & 47 \\
hsa04360 & Axon guidance & $1.07 \times 10^{-05}$ & 43 \\
hsa04022 & cGMP-PKG signaling pathway & $4.12 \times 10^{-05}$ & 39 \\
hsa04972 & Pancreatic secretion & $6.59 \times 10^{-05}$ & 27 \\
hsa04015 & Rap1 signaling pathway & 0.000102002 & 45 \\
hsa04921 & Oxytocin signaling pathway & 0.000156228 & 35 \\
hsa04713 & Circadian entrainment & 0.00019243 & 25 \\
hsa05205 & Proteoglycans in cancer & 0.00021282 & 43 \\
hsa04270 & Vascular smooth muscle contraction & 0.000221981 & 31 \\
\hline
\end{tabular}
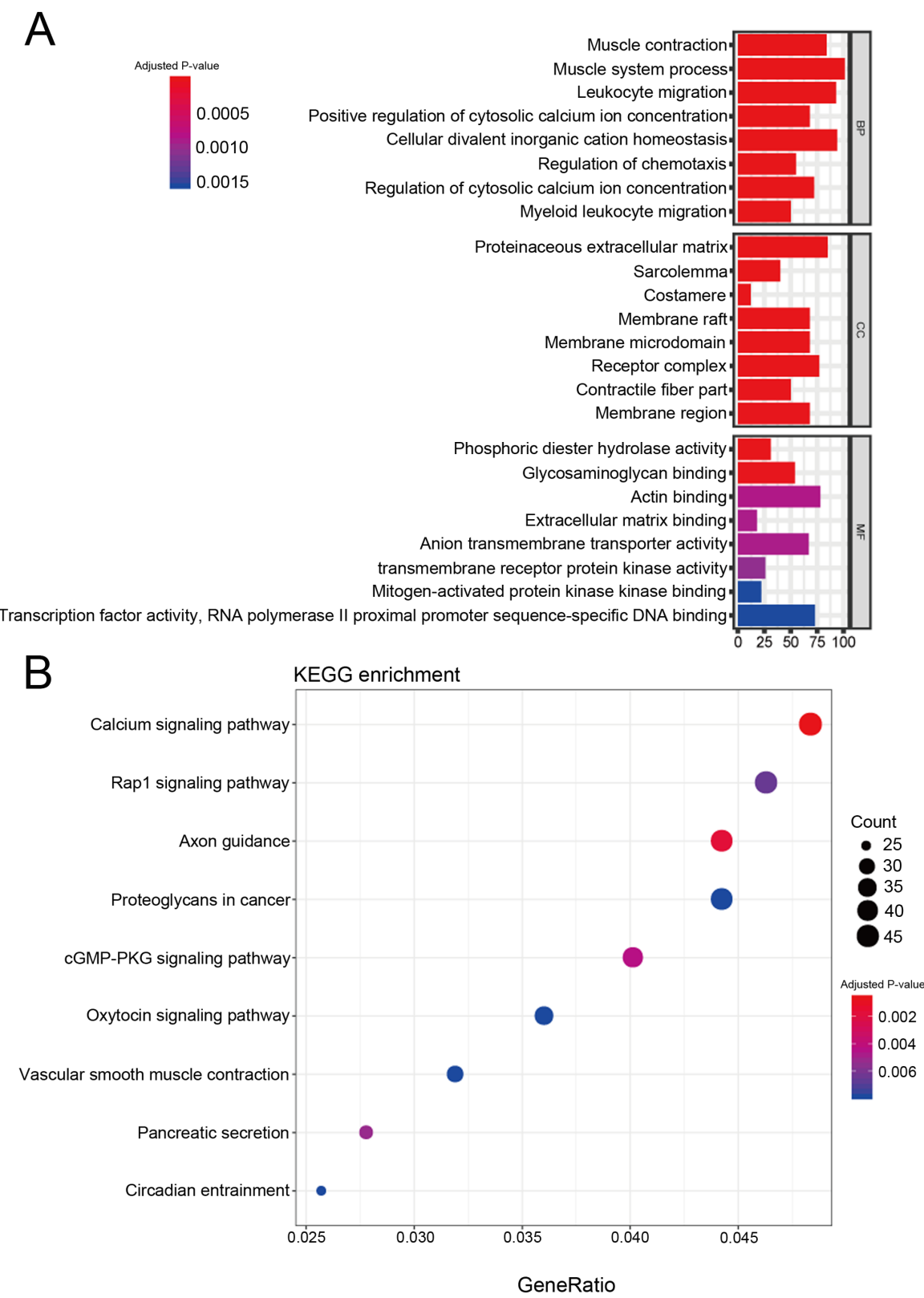

Figure 3. Functional enrichment analysis plots. (A) GO items barplot. The bars represent the enrichment of differentially expressed mRNAs, with vertical axis for GO items and horizontal axis for the number of genes enriched in the items, and the color of the bar is associated with the P-value. (B) KEGG pathway dotplot. The size of the circle represents the number of genes enriched in the pathway, and the color of the circle is associated with the P-value. GO, Gene Ontology; CC, cellular component; MF, molecular function; BP, biological process; KEGG, Kyoto Encyclopedia of Genes and Genomes. 
Table V. DE long non-coding RNAs interacting with the 16 DEmiRNAs retrieved from the ENCORI database.

\begin{tabular}{ll}
\hline IncRNA & \multicolumn{1}{c}{ miRNAs } \\
\hline KCNQ1OT1 & hsa-miR-152-3p, hsa-miR-148a-3p, hsa-miR-29c-3p, hsa-miR-29b-3p, hsa-miR-326 \\
MIR17HG & hsa-miR-130a-3p, hsa-miR-454-3p, hsa-miR-301a-3p, hsa-miR-130b-3p \\
SNHG1 & hsa-miR-326, hsa-miR-421, hsa-miR-21-5p \\
MAGI2-AS3 & hsa-miR-374b-5p, hsa-miR-374a-5p \\
HAGLR & hsa-miR-125a-3p \\
SNHG20 & hsa-miR-495-3p \\
SNHG15 & hsa-miR-24-3p
\end{tabular}

DE, differentially expressed; miRNA/miR, microRNA.

Table VI. List of three types of RNAs involved in the lncRNA-miRNA-mRNA competitive endogenous RNA network.

\begin{tabular}{lll}
\hline RNA & \multicolumn{1}{c}{ Upregulated } & \multicolumn{1}{c}{ Downregulated } \\
\hline lncRNA & MIR17HG, SNHG15, SNHG20, SNHG1, KCNQ1OT1 & HAGLR, MAGI2-AS3 \\
miRNA & hsa-miR-130a-3p, hsa-miR-130b-3p, hsa-miR-148a-3p, \\
& hsa-miR-152-3p, hsa-miR-21-5p, hsa-miR-24-3p, & \\
& hsa-miR-29b-3p, hsa-miR-29c-3p, hsa-miR-301a-3p, & \\
& hsa-miR-374a-5p, hsa-miR-374b-5p, hsa-miR-421, & \\
& hsa-miR-454-3p, hsa-miR-495-3p & \\
mRNA & TOMM34, SCML1, TRAF5, TRIB3, SLC7A5, MET, STX1A, & NTN1, MYLK, CYBRD1, TNS1, \\
& RBM28, RPL28, COL7A1, ABCB6, AMPD2, TMEM9, & CDC14A, EPB41L3, CAV1, TLE4, \\
& SLC6A6, AGAP3, ARHGEF19, ARHGAP39, HOMER1, & TBC1D9, RIPOR2, UST, FAM46A, \\
& C16orf59, VSNL1, ABLIM2, PITX2, CMTM8, BCL2L1, & PDE4D, NR3C1, AKT3, ADGRL2, \\
& SNTB1, SHMT2, KLHL17, ZDHHC9, ADAT2, & PPP1R3C, RECK, SGPP1, FGF13, ZSWIM6, \\
& ANKRD13B & APC, PI15, TTLL7, LYST, MEIS1, LHFPL2, \\
& & FOXO1, DIP2C, DST, ADAMTSL3, PLPP3, \\
& & EDIL3, ZEB2, PCDH7, DMXL1, FLNA, \\
& & LPAR1, L1CAM, DMD, VAMP2
\end{tabular}

lncRNA, long non-coding RNA; miRNA/miR, microRNA.

\section{Discussion}

$\mathrm{CRC}$ is the result of multiple etiological effects, one of which is the assemblage of diverse genetic or epigenetic alterations and their complex connections (21-23). Accumulating evidence has demonstrated that lncRNAs have considerable biological roles by modulating gene expression at multiple levels in tumorigenesis and tumor progression (24), and lncRNAs also form the main part of the ceRNA network of CRC.

Although a number of studies have revealed that the lncRNA-mediated ceRNA network of CRC provides new clues and directions for tumor diagnosis and treatment $(15,25,26)$, to the best of our knowledge, no studies have focused on rectosigmoid junction cancer. The present study first systematically screened DElncRNAs, DEmiRNAs and DEmRNAs, and then successfully constructed a lncRNA-mediated ceRNA network to explore the regulatory mechanism. Functional KEGG enrichment analysis revealed the potential role of DEmRNAs in the carcinogenesis and development of rectosigmoid junction cancer. Furthermore, a few significant RNAs from the ceRNA network were identified, which may represent novel crucial prognostic elements and possible therapeutic targets for rectosigmoid junction cancer.

To the best of our knowledge, no studies have been performed to predict the prognostic value of lncRNAs and their corresponding ceRNA network in rectosigmoid junction cancer. In the present study, 98 DElncRNAs were screened in rectosigmoid junction cancer samples and compared with normal samples, 7 of which were associated with the construction of the ceRNA network. Moreover, it was noted that the upregulated 1ncRNAs KCNQ1OT1 and MIR17HG were closely associated with most of the DEmiRNAs in the ceRNA network. Upregulation of both MIR17HG and KCNQ1OT1 has been previously associated with CRC (27-29), which is consistent with the present results. Upregulated MIR17HG expression promotes tumorigenesis and metastasis in CRC via miR-17-5p to negatively regulate B-cell linker protein, which 


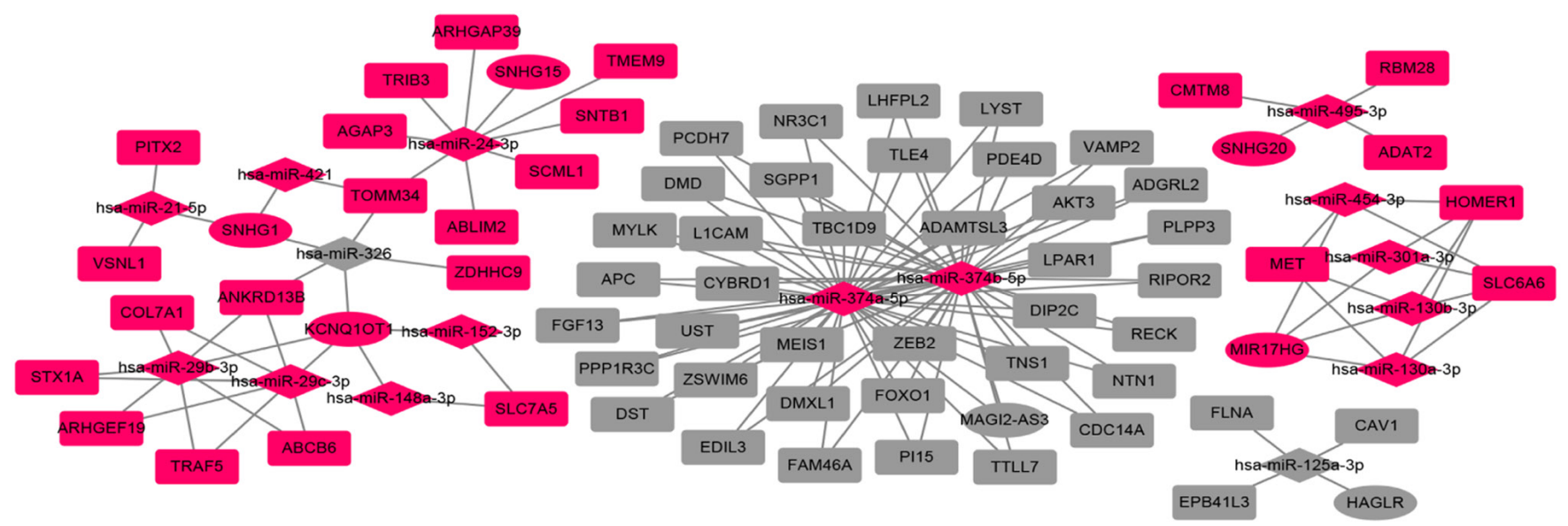

Figure 4. IncRNA-miRNA-mRNA ceRNA network. Circles indicate lncRNAs, diamonds indicate miRNAs and squares indicate mRNAs. Red represents upregulated genes and grey represents downregulated genes. IncRNA, long non-coding RNA; miRNA/miR, microRNA; ceRNA, competitive endogenous RNA.
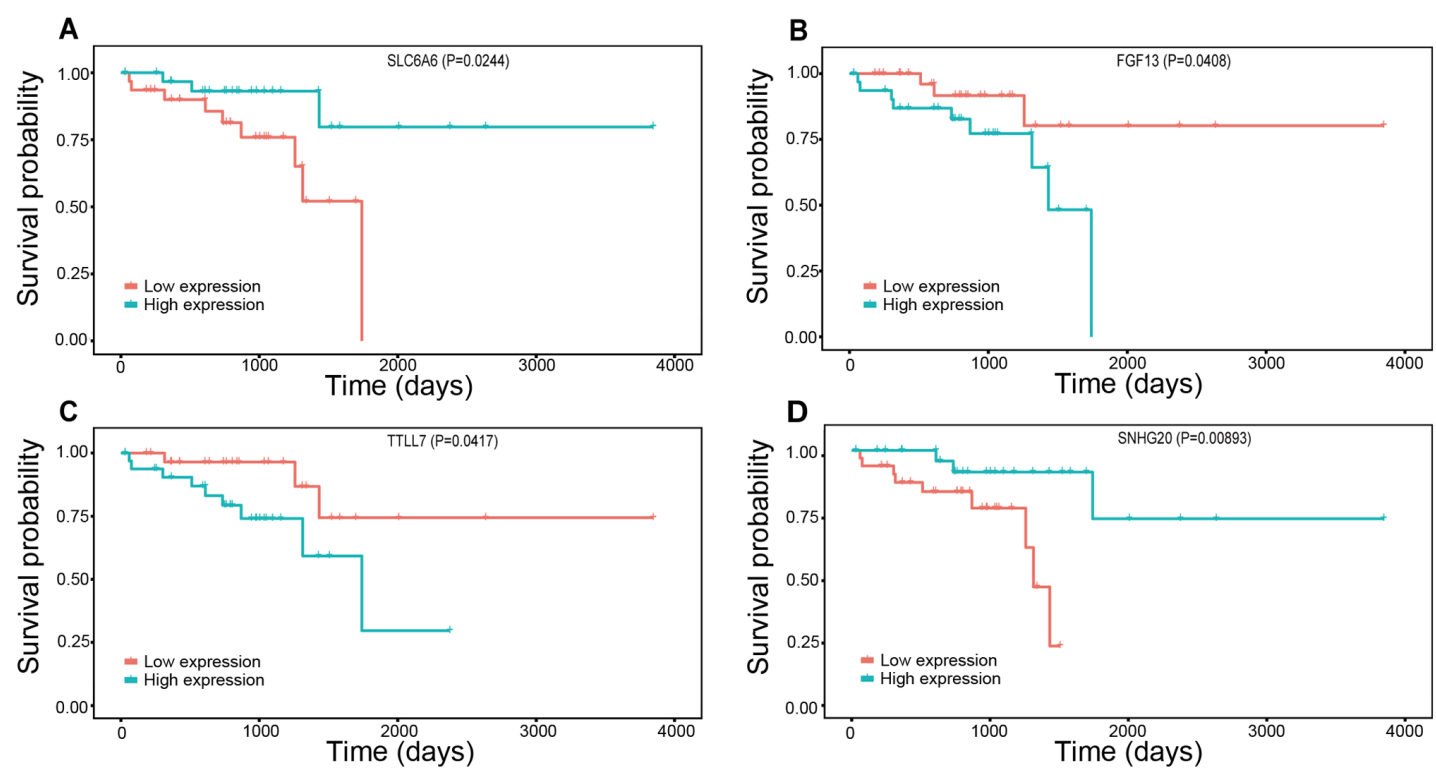

Figure 5. Survival curves of the OS based on the expression levels of three DEmRNAs and one DE long non-coding cRNA in the competitive endogenous RNA network of rectosigmoid junction cancer. (A) SLC6A6, (B) FGF13, (C) TTLL7 and (D) SNHG20. The x-axis indicates the OS time and the y-axis indicates the cumulative survival rate. OS, overall survival; DE, differentially expressed; SNHG20, small nucleolar RNA host gene 20; SLC6A6, sodium- and chloride-dependent taurine transporter; FGF13, fibroblast growth factor 13; TTLL7, tubulin polyglutamylase TTLL7.

also participates in positive feedback regulation through a ceRNA mechanism involving miR-375 in CRC (27).

The dysregulation of KCNQ1OT1 in the lncRNA-coated territory is due to the excessive expression of nuclear $\beta$-catenin, which prompts variation in its lncRNA-coated territory profile; this may trigger a chain reaction that results in the formation of malignant colorectal tumors (28). The present study noted that upregulated KCNQ1OT1 may compete with five key DEmRNAs (miR-152-3p, miR-148a-3p, miR-29c-3p, miR-29b-3p and miR-326) to regulate the expression of target genes. Moreover, high SNHG20 expression resulted in an improved OS in patients with rectosigmoid junction cancer compared with low expression, which was also one of the key genes in the present ceRNA network. As a member of lncRNAs, SNHG20 is frequently expressed in multiple types of tumor, such as hepatocellular carcinoma, ovarian carcinoma, bladder carcinoma and CRC, accounting for tumor development and progression through modifying transcription or post-transcription (30). A previous study revealed that SNHG20 may regulate CRC cell proliferation, invasion and migration through modulation of cyclin A1 and p21 expression (31).

Although lncRNAs have become the focus of research in recent years, miRNAs have also attracted attention. In order to screen the key miRNAs participating in the regulatory network, closeness centrality, degree, MCC and betweenness centrality of all DERNAs were calculated using the cytoHubba plugin on Cytoscape. The results indicated that hsa-miR-374a-5p and hsa-miR-374b-5p were the top two miRNAs ranked among all four calculation methods, suggesting that these two miRNAs with the highest node score may serve a key role in the carcinogenesis and development of rectosigmoid junction cancer. 
Table VII. Node scores of top 10 competitive endogenous RNAs calculated with the cytoHubba plugin on Cytoscape.

\begin{tabular}{ll} 
A, Closeness centrality & \\
\hline RNA & Score \\
\hline hsa-miR-374b-5p & 39.5 \\
hsa-miR-374a-5p & 39.5 \\
MEIS1 & 21 \\
TTLL7 & 21 \\
RECK & 21 \\
PLPP3 & 21 \\
ZSWIM6 & 21 \\
DMD & 21 \\
APC & 21 \\
PCDH7 & 21 \\
\hline
\end{tabular}

\begin{tabular}{lr} 
B, Degree & \\
\hline RNA & Score \\
\hline hsa-miR-374b-5p & 39 \\
hsa-miR-374a-5p & 39 \\
hsa-miR-24-3p & 9 \\
hsa-miR-29c-3p & 7 \\
hsa-miR-29b-3p & 7 \\
hsa-miR-326 & 5 \\
KCNQ1OT1 & 5 \\
MIR17HG & 4 \\
hsa-miR-130a-3p & 4 \\
hsa-miR-454-3p & 4 \\
\hline
\end{tabular}

C, Maximal Clique Centrality

\begin{tabular}{lr} 
RNA & Score \\
\hline hsa-miR-374b-5p & 39 \\
hsa-miR-374a-5p & 39 \\
hsa-miR-24-3p & 9 \\
hsa-miR-29c-3p & 7 \\
hsa-miR-29b-3p & 7 \\
hsa-miR-326 & 5 \\
KCNQ1OT1 & 5 \\
MIR17HG & 4 \\
hsa-miR-130a-3p & 4 \\
hsa-miR-454-3p & 4
\end{tabular}

D, Betweenness centrality

\begin{tabular}{lc}
\hline RNA & Score \\
\hline hsa-miR-374b-5p & 741.0 \\
hsa-miR-374a-5p & 741.0 \\
hsa-miR-326 & 456.7 \\
hsa-miR-24-3p & 376.0 \\
TOMM34 & 356.0
\end{tabular}

Table VII. Continued.

$\mathrm{D}$, Betweenness centrality

\begin{tabular}{ll}
\hline RNA & Score \\
\hline KCNQ1OT1 & 270.3 \\
SNHG1 & 164.0 \\
hsa-miR-29c-3p & 122.7 \\
hsa-miR-29b-3p & 122.7 \\
ANKRD13B & 119.3 \\
\hline
\end{tabular}

miR, microRNA.

Aberrantly expressed miRNAs have been reported to serve a variety of roles in carcinomas. A previous study replicated CRC-specific miRNAs to evaluate prognostic indications, revealing that hsa-miR-374a-5p significantly decreased the risk of death for all cases, regardless of tumor site (32). Additionally, another comprehensive analysis for miRNA expression in breast cancer demonstrated that increased expression levels of hsa-miR-374b-5p and another 11 miRNAs predicted improved breast cancer survival independently (33). However, the present results revealed that these two highly expressed miRNAs resulted in neither improved nor worse survival compared with those without expression. To the best of our knowledge, no studies have identified whether these miRNAs exert functions in tumorigenesis and interact with the prognosis of patients with rectosigmoid junction cancer.

To further understand the biological roles and potential mechanisms of DEmRNAs in the pathogenesis of rectosigmoid junction cancer, KEGG pathway analysis was performed. Among the significant KEGG pathways, 'Proteoglycans in cancer' was the only cancer-associated pathway. The genes caveolin-1 (CAV-1), MET, filamin-A (FLNA) and AKT3 were enriched in the KEGG pathway analysis, as well as being involved in the ceRNA network. Observations from several studies have demonstrated that CAV-1 can exert dual bioactivity, where it acts as a negative regulator in the early stages of CRC but as an oncogene in advanced disease $(34,35)$. Additionally, miR-384 inhibits the proliferation of CRC by directly targeting AKT3 (36). A previous study revealed that FLNA may serve protective roles as a suppressor in CRC SW480 cells by stimulating proliferation via the activation of several signaling pathways (37). Furthermore, another study demonstrated that proteoglycans and glycoproteins act as signal co-receptors or bridging molecules and activate c-MET, which contributes to cancer progression (38). In the present study, MET was enriched in the 'Proteoglycans in cancer' pathway. Notably, it was found that three DEmRNAs (SLC6A6, FFGF13 and TTLL7) involved in the ceRNA network were significantly associated with the OS of patients with rectosigmoid junction cancer $(\mathrm{P}<0.05)$. TTLL7 is a mammalian $\beta$-tubulin polyglutamylase (39), and the phosphinic acid-based inhibitors of TTLL7 are well-characterized inhibitors of polyglutamylases (40), while the modified polyglutamylation is associated with tumorigenesis and resistance to chemotherapeutic drugs targeting microtubules (41). 
In conclusion, the present study identified differentially expressed lncRNAs, miRNAs, and mRNAs that may be used as novel important prognostic biomarkers and potential treatment targets for rectosigmoid junction cancer. A lncRNA-associated ceRNA network was constructed to provide novel and available options for subsequent functional studies of lncRNAs in rectosigmoid junction cancer.

\section{Acknowledgements}

Not applicable.

\section{Funding}

The present study was supported by grants from the Liaoning Province Nature Science Foundation of China (grant no. 2019-ZD-0917) and the Basic Research Program of Higher Education of Liaoning Province of China (grant no. LQ2017035).

\section{Availability of data and materials}

The datasets used and/or analyzed during the current study are available from the corresponding author on reasonable request.

\section{Authors' contributions}

QZ and SR designed the study. YZ, ZF and SS also participated in the design of this study and made suggestions in the research process. SS and ZF acquired and downloaded the data from databases. QZ and YZ performed the bioinformatics analyses. QZ and ZF wrote the manuscript. QZ, SS and ZF reviewed and edited manuscripts. SR and YZ supervised the whole project. All authors read and approved the final manuscript.

\section{Ethics approval and consent to participate}

Not applicable.

\section{Patient consent for publication}

Not applicable.

\section{Competing interests}

The authors declare that they have no competing interests.

\section{References}

1. Lee YC, Lee YL, Chuang JP and Lee JC: Differences in survival between colon and rectal cancer from SEER data. PLoS One 8: e78709, 2013.

2. Bray F, Ferlay J, Soerjomataram I, Siegel RL, Torre LA and Jemal A: Global cancer statistics 2018: GLOBOCAN estimates of incidence and mortality worldwide for 36 cancers in 185 countries. CA Cancer J Clin 68: 394-424, 2018.

3. World Health Organization: International classification of diseases for oncology (ICD-O)-3rd edition, 1st revision, 2013 https://apps.who.int/iris/handle/10665/96612

4. D'Souza N, de Neree Tot Babberich MPM, Lord A, Shaw A, Abulafi M, Tekkis P, Wiggers T and Brown G: The rectosigmoid problem. Surg Oncol 27: 521-525, 2018.
5. Falch C, Mueller S, Braun M, Gani C, Fend F, Koenigsrainer A and Kirschniak A: Oncological outcome of carcinomas in the rectosigmoid junction compared to the upper rectum or sigmoid colon-A retrospective cohort study. Eur J Surg Oncol 45: 2037-2044, 2019.

6. Käser SA, Froelicher J, Li Q, Müller S, Metzger U, Castiglione M, Laffer UT and Maurer CA: Adenocarcinomas of the upper third of the rectum and the rectosigmoid junction seem to have similar prognosis as colon cancers even without radiotherapy, SAKK 40/87. Langenbecks Arch Surg 400: 675-682, 2015 .

7. Ponz de Leon M, Marino M, Benatti P, Rossi G, Menigatti M, Pedroni M, Di Gregorio C, Losi L, Borghi F, Scarselli A, et al: Trend of incidence, subsite distribution and staging of colorectal neoplasms in the 15-year experience of a specialised cancer registry. Ann Oncol 15: 940-946, 2004.

8. Bhan A, Soleimani M and Mandal SS: Long noncoding RNA and cancer: A new paradigm. Cancer Res 77. 15: 3965-3981, 2017.

9. Rupaimoole R and Slack FJ: MicroRNA therapeutics: Towards a new era for the management of cancer and other diseases. Nat Rev Drug Discov 16: 203-222, 2017.

10. Sergeeva OV, Koteliansky VE and Zatsepin TS: mRNA-based therapeutics-advances and perspectives. Biochemistry (Mosc) 81: 709-722, 2016.

11. Salmena L, Poliseno L, Tay Y, Kats L and Pandolfi PP: A ceRNA hypothesis: The Rosetta Stone of a hidden RNA language? Cell 146: 353-358, 2011.

12. Cheng Y, Geng L, Wang K, Sun J, Xu W, Gong S and Zhu Y: Long noncoding RNA expression signatures of colon cancer based on the ceRNA network and their prognostic value. Dis Markers 2019: 7636757, 2019.

13. Luo R, Song J, Zhang W and Ran L: Identification of MFI2-AS1, a novel pivotal lncRNA for prognosis of stage III/IV colorectal cancer. Dig Dis Sci 65: 538-3550, 2020.

14. Wang L, Cho KB, Li Y, Tao G, Xie Z and Guo B: Long noncoding RNA (lncRNA)-mediated competing endogenous RNA networks provide novel potential biomarkers and therapeutic targets for colorectal cancer. Int J Mol Sci 20: 5758, 2019.

15. Zhang Z, Wang S, Ji D, Qian W, Wang Q, Li J, Gu J, Peng W, $\mathrm{Hu} \mathrm{T}$, Ji B, et al: Construction of a ceRNA network reveals potential lncRNA biomarkers in rectal adenocarcinoma. Oncol Rep 39: 2101-2113, 2018.

16. Dong X, Yang Z, Yang H, Li D and Qiu X: Long Non-coding RNA MIR4435-2HG promotes colorectal cancer proliferation and metastasis through miR-206/YAP1 Axis. Front Oncol 10: $160,2020$.

17. Zhang X, Wen L, Chen S, Zhang J, Ma Y, Hu J, Yue T, Wang J, Zhu J, Bu D and Wang X: The novel long noncoding RNA CRART16 confers cetuximab resistance in colorectal cancer cells by enhancing ERBB3 expression via miR-371a-5p. Cancer Cell Int 20: 68, 2020.

18. Amin MB, Edge S, Greene F, Byrd DR, Brookland RK, Washington MK, Gershenwald JE, Compton CC, Hess KR, Sullivan DC, Jessup JM, Brierley JD, Gaspar LE, Schilsky RL, Balch CM, Winchester DP, Asare EA, Madera M, Gress DM, Meyer LR (eds.): AJCC Cancer Staging Manual. 8th edition. Springer, 2017.

19. Chou CH, Shrestha S, Yang CD, Chang NW, Lin YL, Liao KW, Huang WC, Sun TH, Tu SJ, Lee WH, et al: miRTarBase update 2018: A resource for experimentally validated microRNA-target interactions. Nucleic Acids Res 46: D296-D302, 2018.

20. Li JH, Liu S, Zhou H, Qu LH and Yang JH: starBase v2.0: Decoding miRNA-ceRNA, miRNA-ncRNA and protein-RNA interaction networks from large-scale CLIP-Seq data. Nucleic Acids Res 42: D92-D97, 2014.

21. Lindner P, Paul S, Eckstein M, Hampel C, Muenzner JK, Erlenbach-Wuensch K, Ahmed HP, Mahadevan V, Brabletz T, Hartmann A, et al: EMT transcription factor ZEB1 alters the epigenetic landscape of colorectal cancer cells. Cell Death Dis 11: 147, 2020.

22. Mo S, Zhang L, Dai W, Han L, Wang R, Xiang W, Wang Z, Li Q, Yu J, Yuan J, et al: Antisense lncRNA LDLRAD4-AS1 promotes metastasis by decreasing the expression of LDLRAD4 and predicts a poor prognosis in colorectal cancer. Cell Death Dis 11: 155, 2020.

23. Stuckel AJ, Zhang W, Zhang X, Zeng S, Dougherty U, Mustafi R, Zhang Q, Perreand E, Khare T, Joshi T, et al: Enhanced CXCR4 expression associates with increased gene body 5 -Hydroxymethylcytosine modification but not decreased promoter methylation in colorectal cancer. Cancers (Basel) 12: 539, 2020. 
24. Kopp F and Mendell JT: Functional classification and experimental dissection of long noncoding RNAs. Cell 172: 393-407, 2018.

25. Liu J, Li H, Zheng B, Sun L, Yuan Y and Xing C: Competitive endogenous RNA (ceRNA) regulation network of lncRNA-miRNA-mRNA in colorectal carcinogenesis. Dig Dis Sci 64: 1868-1877, 2019.

26. Wei S, Chen J, Huang Y, Sun Q, Wang H, Liang X, Hu Z and Li X Identification of hub genes and construction of transcriptional regulatory network for the progression of colon adenocarcinoma hub genes and TF regulatory network of colon adenocarcinoma. J Cell Physiol 235: 2037-2048, 2020.

27. Xu J, Meng Q, Li X, Yang H, Xu J, Gao N, Sun H, Wu S, Familiari G, Relucenti M, et al: Long noncoding RNA MIR17HG promotes colorectal cancer progression via miR-17-5p. Cancer Res 79: 4882-4895, 2019.

28. Sunamura N, Ohira T, Kataoka M, Inaoka D, Tanabe $H$ Nakayama Y, Oshimura M and Kugoh H: Regulation of functional KCNQ1OT1 lncRNA by beta-catenin. Sci Rep 6: 20690, 2016.

29. Li F, Li Q and Wu X: Construction and analysis for differentially expressed long non-coding RNAs and MicroRNAs mediated competing endogenous RNA network in colon cancer. PLoS One 13: e0192494, 2018.

30. Zhao W, Ma X, Liu L, Chen Q, Liu Z, Zhang Z, Ma S, Wang Z, Li H, Wang Z and Wu J: SNHG20: A vital lncRNA in multiple human cancers. J Cell Physiol: Jan 15, 2019 (Epub ahead of print). doi: $10.1002 /$ jep. 28143 .

31. Li C, Zhou L, He J, Fang XQ, Zhu SW and Xiong MM: Increased long noncoding RNA SNHG20 predicts poor prognosis in colorectal cancer. BMC Cancer 16: 655, 2016.

32. Slattery ML, Herrick JS, Mullany LE, Valeri N, Stevens J, Caan BJ, Samowitz W and Wolff RK: An evaluation and replication of miRNAs with disease stage and colorectal cancer-specific mortality. Int J Cancer 137: 428-438, 2015.
33. Chang JT, Wang F, Chapin W and Huang RS: Identification of MicroRNAs as breast cancer prognosis markers through the cancer genome atlas. PLoS One 11: e0168284, 2016.

34. Torrejon B, Cristobal I, Rojo F and Garcia-Foncillas J: Caveolin-1 is markedly downregulated in patients with early-stage colorectal cancer. World J Surg 41: 2625-2630, 2017.

35. Yang J, Zhu T, Zhao R, Gao D, Cui Y, Wang K and Guo Y: Caveolin-1 inhibits proliferation, migration, and invasion of human colorectal cancer cells by suppressing phosphorylation of epidermal growth factor receptor. Med Sci Monit 24: 332-341, 2018.

36. Wang YX, Zhu HF, Zhang ZY, Ren F and Hu YH: MiR-384 inhibits the proliferation of colorectal cancer by targeting AKT3. Cancer Cell Int 18: 124, 2018

37. Wang K, Zhu TN and Zhao RJ: Filamin A regulates EGFR/ERK/Akt signaling and affects colorectal cancer cell growth and migration. Mol Med Rep 20: 3671-3678, 2019.

38. Noriega-Guerra $\mathrm{H}$ and Freitas VM: Extracellular matrix influencing HGF/c-MET signaling pathway: Impact on cancer progression. Int J Mol Sci 19: 3300, 2018.

39. Ikegami K, Mukai M, Tsuchida J, Heier RL, Macgregor GR and Setou M: TTLL7 is a mammalian beta-tubulin polyglutamylase required for growth of MAP2-positive neurites. J Biol Chem 281: 30707-30716, 2006.

40. Liu Y, Garnham CP, Roll-Mecak A and Tanner ME: Phosphinic acid-based inhibitors of tubulin polyglutamylases. Bioorg Med Chem Lett 23: 4408-4412, 2013.

41. Das V, Kanakkanthara A, Chan A and Miller JH: Potential role of tubulin tyrosine ligase-like enzymes in tumorigenesis and cancer cell resistance. Cancer Lett 350: 1-4, 2014.

This work is licensed under a Creative Commons Attribution-NonCommercial-NoDerivatives 4.0 International (CC BY-NC-ND 4.0) License. 